\title{
Prevalence of inducible clindamycin resistance in erythromycin resistant clinical isolates of Staphylococcus aureus and CONS at tertiary care hospital
}

\author{
Sangita Thapa, ${ }^{1}$ Lokendra Bahadur Sapkota ${ }^{2}$ \\ ${ }^{1}$ Department of Clinical Microbiology \& Immunology, ${ }^{2}$ Department of Biochemistry, Chitwan Medical Col- \\ lege, Bharatpur-10, Chitwan, Nepal.
}

\section{Correspondence \\ Dr. Sangita Thapa \\ Department of Clinical \\ Microbiology \& \\ Immunology, Chitwan \\ Medical College, Bharatpur- \\ 10, Chitwan, Nepal.}

\section{Email:}

drsangitathapa@gmail.com

DOI: http://

dx.doi.org/10.3126/

jemsn.v12i3.16011

Article received: July $30^{\text {th }}$ 2016

Article accepted: $\operatorname{Aug} 22^{\text {nd }}$

2016

\begin{abstract}
Background \& Objectives: The objective of this study was to isolate and identify Staphylococcus species from different samples clinical samples and to determine the current trend regarding the incidence and distribution of inducible clindamycin resistance in clinical isolates of Staphylococcus aureus and CONS. Materials \& Methods: A total of 264 isolates of staphylococcus species were isolated from various clinical samples. Clinical samples were cultured and Staphylococcus species were identified using standard microbiological methods recommended by the American Society for Microbiology (ASM). Methicillin resistance was confirmed using cefoxitin and oxacillin disks. Inducible clindamycin resistance was identified using D-zone test. Results: Among 264 erythromycin resistant staphylococcus species, $213(80.6 \%)$ were S. aureus and $51(19.3 \%)$ were CONS. Out of 213 erythromycin resistant isolates of S. aureus, 140 $(65.7 \%)$ were MRSA and $73(34.2 \%)$ were MSSA whereas out of 51 erythromycin resistant isolates of CONS, 28 (54.9\%) were MRCNS and 23 (45\%) were MSCNS. Constitutive MLSB phenotype and Inducible MLSB phenotype was higher among both MRSA and MRCNS isolates. MS phenotype was more predominant among $11(5.1 \%)$ MSSA and $5(9.8 \%)$ MSCNS isolates compared to $9(4.2 \%)$ in MRSA and $2(3.9 \%)$ in MRCNS. Conclusion: The prevalence of constitutive \& inducible clindamycin resistance in staphylococcus isolates was high among both MRSA and MRCNS. Hence the implementation of D-test routinely, will reveal the iMLSB \& cMLSB phenotype \& will guide the clinicians whether to use clindamycin in staphylococcal infections when erythromycin resistance is present.
\end{abstract}

Key words: Clindamycin; D-test; Erythromycin; MRSA; Staphylococcus species.

Citation: Thapa S, Sapkota LB. Prevalence of inducible clindamycin resistance in erythromycin resistant clinical isolates of Staphylococcus aureus and CONS at tertiary care hospital. JCMS Nepal. 2016;12(3):83-8.

\section{INTRODUCTION}

Staphylococcus aureus and Coagulase Negative Staphylococci (CONS) are recognized to be most common cause of nosocomial and community acquired infections in every region of the world. The increasing prevalence of Methicillin resistant among staphylococci is a global problem. ${ }^{1}$ Methicillin-resistant Staphylococcus aureus (MRSA) are increasingly being reported as multidrug resistant with high resistance to most of the commonly used antimicrobial agents like; macrolides (erythromycin, clarithromycin), lincosamides (clindamycin, lincomycin), aminoglycosides, chloramphenicol, tetracycline and fluoroquinolones, leaving very few therapeutic options. ${ }^{2}$ In addition, MRSA strains should be considered to be resistant to all cephalosporins, cephems and other beta-lactams (such as ampicillin sulbactam, amoxicillin clavulanic acid, ticarcillinclavulanic acid, piperacillin-tazobactam and the carbapenems) regardless of the in vitro test results obtained with those agents. ${ }^{3}$ Newer antibiotics like vancomycin, linezolid, and quinupristin-dalfopristin have been advocated in the management of these 
isolates, but recent reports of resistance to these agents raise real concerns over how long these uniform susceptibilities will hold good. ${ }^{4,5}$ This has led to renewed interest in the usage of macrolide (e.g., erythromycin)-lincosamide (e.g., Clindamycin)-streptogramin B (e.g., quinupristindalfopristin) (collectively called as MLSB family) antibiotics to treat Staphylococcus aureus (S. aureus) infections with, clindamycin being the preferred agent due to its excellent pharmacokinetic properties. Staphylococcal strains resistant to MLSB antibiotics have increased in number following the widespread use of these antibiotics for treating serious staphylococcal infections. ${ }^{6}$

Resistance occurs by different mechanisms to these microbiologically related antibiotics. Resistance due to active efflux encoded by msr (A) gene confers resistance to macrolides and streptogramin B (MS phenotype) but not to clindamycin. Ribosomal target modification, another mechanism of resistance, confers resistance to macrolide, type B streptogramin and also to clindamycin (MLSB phenotype). MLSB resistance in staphylococci is either constitutive (c), where rRNA methylase is always produced or inducible (iMLSB), where methylase is only produced in the presence of an inducer, and is encoded by erm (A) or erm (C) gene. $^{7,8}$ The resistance is constitutive (cMLSB) when R-methylase is produced and inducible (iMLSB) (MLSB)when methylase is produced only in the presence of an inducing agent. Erythromycin is a very effective inducer and Clindamycin is a weak inducer. ${ }^{9}$ Patients infected with iMLSB (MLSB)strains of staphylococcus if treated with clindamycin can develop constitutive resistance during therapy and subsequently result in treatment failure. $^{10}$

Detection of three resistant phenotypes (MS, iMLSB, cMLSB) is crucial for guiding appropriate antimicrobial therapy. Constitutive resistance can be detected by routine disc diffusion method but it fails to detect inducible resistance (iMLSB), which appears sensitive to clindamycin on routine testing, resulting in institution of inappropriate clindamycin therapy. Inducible resistance also cannot be detected by broth or agar dilution methods. ${ }^{11}$ Double disc diffusion (D test) is recommended by CLSI for detection of inducible clindamycin resistance. A negative result for inducible clindamycin resistance (ICR) by D test confirms clindamycin susceptibility and provides a good therapeutic option, thus necessitates the detection of inducible clindamycin resistance. $^{12}$

Incidence of clindamycin resistance varies from place to place and therefore a local data is important to guide empirical treatment. ${ }^{13}$ Data describing prevalence of clindamycin resistance among clinical isolates of S. aureus and CONS is lacking from our geographic area. So this study demonstrates simple, reliable and significant method (double disc diffusion test) of detecting inducible resistance to Clindamycin in isolates of S. aureus and CONS.

\section{MATERIALS AND METHODS}

Methods: A prospective study was conducted from March 2015 to December 2015 at Chitwan Medical College Teaching Hospital (a 600 bed teaching hospital), Chitwan, Nepal.

Sample collection: The samples were collected in sterile containers using aseptic technique and transported to the laboratory without delay. All samples were processed immediately. A total of 264 non duplicate clinical isolates of erythromycin resistant Staphylococcus species isolated from samples received from various outpatient and inpatient departments of the hospital were included in the study.

Culture and bacterial identification: For the isolation and identification of Staphylococcus species several media used were blood agar (BA), chocolate agar (CHA), MacConkey agar (MA), brain heart infusion (BHI) broth (for blood sample), DNase agar and mannitol salt agar (HiMedia Laboratories Pvt. Limited, India) and the tests used were catalase and coagulase. The collected samples were inoculated onto different culture media. The CHA plates were incubated in a $\mathrm{CO} 2$ incubator $(10 \% \mathrm{CO} 2)$ at $37^{\circ} \mathrm{C}$ for 24 hours. The BA and MA plates were incubated at $37^{\circ} \mathrm{C}$ for 24 hours in an aerobic atmosphere. Staphylococcus species were identified by standard microbiological techniques. 14

Antibiotic susceptibility testing: Antibiotic susceptibility test was performed by modified Kirby -Bauer disk diffusion method on Mueller- Hinton agar following Clinical and Laboratory Standards Institute (CLSI) guidelines. Antibiotic disks (HiMedia Laboratories, Pvt. Limited, India) used were: penicillin $\mathrm{G}(10 \mathrm{U})$, ciprofloxacin $(5 \mu \mathrm{g})$, erythromycin $(15 \mu \mathrm{g})$, co-trimoxazole $(25 \mu \mathrm{g})$, gentamicin $(10 \mu \mathrm{g})$, amikacin $(30 \mu \mathrm{g})$, cephalexin $(30 \mu \mathrm{g})$, ceftriaxone $30 \mu \mathrm{g})$, cefoxitin $(30 \mu \mathrm{g})$, oxacillin $(1 \mu \mathrm{g})$, vancomycin $(30 \mu \mathrm{g})$, clindamycin 
$(2 \mu \mathrm{g})$ and teicoplanin $(30 \mu \mathrm{g})$.

Identification of methicillin resistant Staphylococcus aureus (MRSA) strains: Methicillin resistant Staphylococcus aureus (MRSA) was identified by using oxacillin $(1 \mu \mathrm{g})$ and cefoxitin $(30 \mu \mathrm{g})$ disks. Plates were incubated at $35^{\circ} \mathrm{C}$. Plates containing oxacillin disk were read following a 24 hours of incubation period. The diameter of the zone of inhibition (ZOI) of growth was recorded and interpreted as susceptible or resistant according to the criteria of CLSI. Staphylococcus aureus isolates were deemed methicillin resistant when the ZOI was $\leq 10 \mathrm{~mm}$ with the oxacillin disk or $\leq 21$ $\mathrm{mm}$ with the cefoxitin disk [15]. For MRSA detection, Staphylococcus aureus ATCC 25923 and ATCC 43300 were used as negative and positive controls respectively.

Detection of inducible clindamycin resistant strains: Inducible macrolide-lincosamidestreptogramin B (iMLSB) resistance was detected by Disk approximation test placing a $2 \mu \mathrm{g}$ clindamycin disk $15 \mathrm{~mm}$ away from the edge of a $15 \mu \mathrm{g}$ erythromycin disk on a MHA plate. Staphylococcus aureus ATCC 25923 was used as a control organism for antibiotic sensitivity testing. Following overnight incubation at $37^{\circ} \mathrm{C}$, three different phenotypes were appreciated and interpreted as follows: ${ }^{16}$

a. Inducible MLS (iMLBS) phenotype $\square$ Staphylococcal isolates showing resistance to erythromycin while being sensitive to clindamycin and giving D $\square$ shaped zone of inhibition around clindamycin with flattening towards erythromycin disc.

b. Constitutive MLS (cMLSB) phenotype - Those Staphylococcal isolates, which showed resistance to both erythromycin and clindamycin with circular shape of zone of inhibition, if any around clindamycin.

c. MS phenotype $\square$ Isolates exhibiting resistance to erythromycin and sensitivity to clindamycin and giving circular zone of inhibition around clindamycin.

\section{RESULTS}

Out of 264 Erythromycin resistant staphylococcus species, 213 were S. aureus and 51 were CONS. Majority of the samples $57.9 \%$ were received from inpatient department and $42 \%$ from outpatient department. Most of the staphylococcus species were found in age group one to 10 years as shown in Figure 1. Staphylococcus species were most commonly isolated form blood sample $44.6 \%$ followed by pus/exudate $23.4 \%$, urine $11.7 \%$, and sputum $10.2 \%$ as shown in Figure 2. Out of 213 Erythromycin resistant isolates of S. aureus, 140 $(65.7 \%)$ were MRSA whereas out of 51 Erythromycin resistant isolates of CONS, 28 (54.9\%) were MRCNS. In this study Constitutive MLSB phenotype was most predominant phenotype $48.4 \%$ followed by Inducible MLSB phenotype $41.2 \%$ and MS phenotype 10.2\% among staphylococcus species. Constitutive MLSB phenotype and Inducible MLSB phenotype was predominant among both MRSA and MRCNS isolates. MS phenotype was more predominant among MSSA and MSCNS isolates as shown in Table 1.

\section{DISCUSSION}

Staphylococcus aureus and CONS can cause wide spectrum of infections from localized to deep seated infections. Most of the of Staphylococcus isolates are showing multidrug resistance to commonly used antimicrobial agents. The emergence of methicillin resistant staphylococcus aureus is a global problem. ${ }^{17}$ Erythromycin is most commonly used drug for treatment of both minor and major serious staphylococcal infections. Due to increasing burden of erythromycin resistance there is very limited therapeutic option left for treatment of staphylococcal infections. Clindamycin is most preferred drug for treatment of MRSA infections. ${ }^{18}$ Macrolide resistant staphylococcus species may show constitutive or inducible resistance to clindamycin or may be resistance to only macrolides. However, there has been increase in inducible clindamycin resistance among erythromycin resistant clinical isolates of staphylococcus species. Staphylococcal species with clindamycin resistance can develop inducible phenotype, and gradually from such isolates, spontaneous constitutively resistant mutants have arisen both in vitro and in vivo during clindamycin therapy. More over negative result for inducible clindamycin resistance confirms clindamycin susceptibility and good therapeutic option for treatment of both MRSA and MSSA. Hence, detection of such resistant phenotypes is of utmost importance to minimize treatment failures. ${ }^{19}$

Maximum (34.4\%) of staphylococcus species were isolated from age group 0 to 10 years . Majority of the staphylococcus species $44.6 \%$ were isolated 


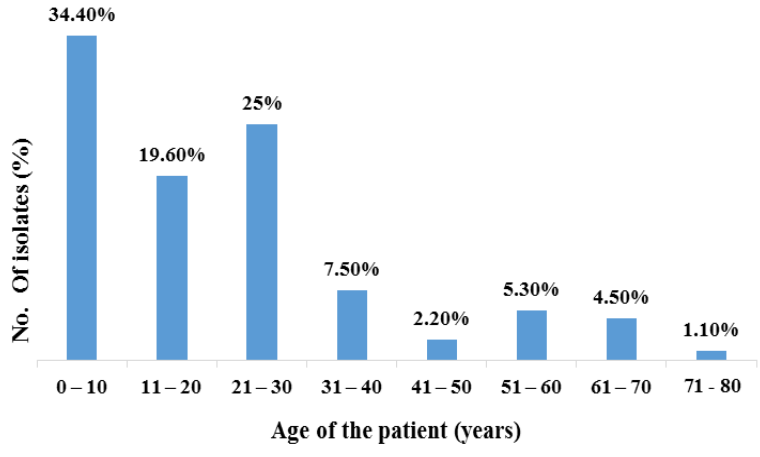

Figure 1: Age wise distribution of clinical isolates of staphylococcus species.

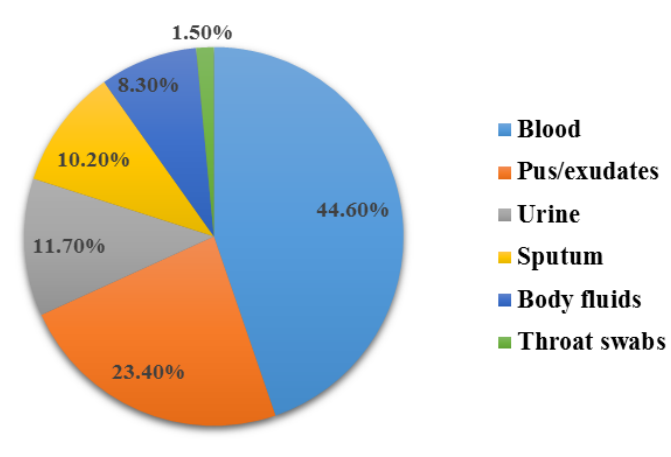

Figure 2: Distribution of staphylococcus species from different clinical samples.

Table 1: Phenotypic variation of isolates according to clindamycin resistance.

$\begin{array}{lccccc}\text { Phenotype } & \text { MRSA } & \text { MSSA } & \text { MRCNS } & \text { MSCNS } & \text { Total } \\ \begin{array}{l}\text { iMLSB (E-R, CD-S, D } \\ \text { test +) }\end{array} & 53 & 36 & 12 & 8 & 109(41.2) \\ \text { cMLSB (E-R, CD-R) } & 78 & 26 & 14 & 10 & 128(48.4) \\ \begin{array}{l}\text { MS phenotype (E-R, CD } \\ \text {-S, D test -) }\end{array} & 9 & 11 & 2 & 5 & 27(10.2) \\ \text { Total } & 140(65.7 \%) & 73(34.2 \%) & 28(54.9 \%) & 23(45 \%) & 264(100 \%)\end{array}$

from blood sample followed by pus/exudates $23.4 \%$ which shows the role of staphylococcus species in bloodstream infections and abscess formation. These findings are in concordance with study conducted by Faisal et al. ${ }^{20}$ Various other investigators reported that majority of staphylococcus species were recovered from pus and urine samples respectively. ${ }^{21,22}$ This shows the variation among staphylococcus isolates recovered from clinical samples in different healthcare setting.

In our study among 264 erythromycin resistant staphylococcus species, $213(80.6 \%)$ were S. aureus and $51(19.3 \%)$ were CONS. In present study Constitutive MLSB phenotype was most predominant phenotype $48.4 \%$ followed by Inducible MLSB phenotype $41.2 \%$ and MS phenotype $10.2 \%$ among staphylococcus species. Various other investigators reported the similar results. ${ }^{23}$ Out of 213 erythromycin resistant isolates of S. aureus, $140(65.7 \%)$ were MRSA and 73 $(34.2 \%)$ were MSSA whereas out of 51 erythromycin resistant isolates of CONS, 28 (54.9\%) were MRCNS and 23 (45\%) were MSCNS. There is high prevalence of Constitutive MLSB phenotype and Inducible MLSB phenotype among both MRSA and MRCNS isolates. Similar pattern has been observed in earlier studies. ${ }^{24,25}$
Also khan et al reported that Constitutive MLSB phenotype and Inducible MLSB phenotype was higher among both MRSA and MRCNS isolates. ${ }^{26}$ MS phenotype was more predominant among 11 (5.1\%) MSSA and 5 (9.8\%) MSCNS isolates compared to $9(4.2 \%)$ in MRSA and $2(3.9 \%)$ in MRCNS which is in concordance with study done by other investigators. ${ }^{27}$

The variation of the results depends upon the sample size, age group, geographical region, population studied and antibiotic profile. The prevalence of inducible clindamycin resistance varies among different hospital setting. So this study was conducted to determine inducible clindamycin resistance in our locality. The present study demonstrates the high prevalence of Constitutive MLSB phenotype and Inducible MLSB phenotype among both MRSA and MRCNS isolates. There is very limited therapeutic option available for treatment of MRSA infections, clindamycin being more preferred than vancomycin. Hence, true sensitivity to clindamycin can be judged by performing simple ' $\mathrm{D}$ ' test for all erythromycin resistant staphylococcus species. Therefore, by performing this simple test routinely clindamycin treatment failure can be greatly prevented. 


\section{CONCLUSION}

Treatment of beta-lactamase producing and methicillin resistant staphylococcal infection are ever challenging. Keeping the mode of action, adverse reactions and pharmacokinetics in mind of certain antibiotics like vancomycin, clindamycin should be preferred for the treatment of severe and resistant infections. Present study gives an information regarding the presence of high percentage of inducible clindamycin resistance among the erythromycin resistant staphylococci. The frequency of inducible clindamycin resistance among Staphylococcus species may differ in different hospital setups. Clinical microbiology laboratories should implement simple and effective testing, D-test on all Staphylococcus species before reporting about the clindamycin susceptibility. Reporting S. aureus and CONS as susceptible to clindamycin without checking for inducible resistance may result in institution of inappropriate clindamycin therapy. On the other hand, negative result for inducible clindamycin resistance confirms clindamycin susceptibility and provides a very good therapeutic option.

\section{Recommendations}

Based on the findings of this study, D- test should be mandatory for all routine microbiological laboratories before reporting about the clindamycin susceptibility for all erythromycin resistant staphylococcus species. Reporting Staphylococcus species as susceptible to clindamycin without checking for inducible resistance may result treatment failure with clindamycin therapy. For D test positive isolates clindamycin is not a suitable drug and for $\mathrm{D}$ negative isolates clindamycin is a good therapeutic option for both MRSA and MSSA isolates.

\section{REFERENCES}

1. Yilmaz G, Ayden k, Iskender S, Caylan R and Koksal I. Detection and prevalence of inducible Clindamycin resistance in staphylococci. Journal of medical microbiology DOI: 10.1099/jmm.0.46761-0. PMID:17314364.

2. Mandell G, Douglas J, Bennett R: Principles and practice of infectious disease. 4th edition. Edinburgh, United Kingdome: Churchill Livingstone Ltd.; 1995.

3. National Committee for Clinical Laboratory Standards (NCCLS): Performance standards for antimicrobial susceptibility testing. 7th edition. Wayne PA, USA: NCCLS; 2000. Vol. 20

4. Johnson AP, Woodford N. Glycopeptide-resistant Staphylococcus aureus. J Atimicrob Chemother.
2002;50:621-23. DOI: 10.1093/jac/dkf244.

5. Eliopoulos GM. Quinupristin-dalfopristin and linezolid: Evidence and opinion. Clin Infect Dis. 2003;6:473-81. DOI: 10.1086/367662. PMID:12567306.

6. Delialioglu N, Aslan G, Ozturk C, Baki V, Sen S, Emekdas G. Inducible clindamycin resistance in staphylococci isolated from clinical samples. Jpn J Infect Dis. 2005;58:104-6. PMID: 15858290.

7. Chelae S, Laohaprertthisarn V, Phengmak M, Kongmuang U, Kalnauwakul S. Detection of inducible clindamycin resistance in staphylococci by disc diffusion induction test. J Med Assoc Thai. 2009;92(7):947-51. PMID:19626815.

8. Leclercq R. Mechanism of resistance to macrolides and lincosamides: nature of the resistance elements and their clinical implications. Clin Infect Dis. 2002;34:482-92. DOI: 10.1086/324626. PMID:11797175.

9. Gupta V, Datta P, Rani H, Chander J. Inducible clindamycin resistance in Staphylococcus aureus: A study from North. J PG Med. 2009;55(3):176-9. DOI: 10.4103/0022-3859.57393.

10. Siberry GK, Tekle T, Carroll k, Dick J. Failure of clindamycin treatment in methicillin resistant Staphylococcus aureus expressing inducible clindamycin resistance in vitro. C Lin Infect Dis. 2003;37:1257-60. DOI: $10.1086 / 377501$. PMID:14557972.

11. Fiebelkorn KR, Crawford SA, McElmeel ML, Jorgensen JH. Practical disk diffusion method for detection of inducible clindamycin resistance in Staphylococcus aureus and coagulase-negative staphylococci. J Clin Microbiol. 2003;41:4740-4. DOI: 10.1128/JCM.41.10.47404744.2003. PMID:14532213.

12. Rodrigues Perez LR, Caierao J, Souza Antunes AL, Alves d'Azevedo P. Use of D test method to detect inducible clindamycin resistance in coagulase negative staphylococci (CoNS). Braz J Infect Dis. 2007;11:186-8.

13. Schreckenberger PC, Ilendo E, Ristow KL. Incidence of constitutive and clindamycin resistance in Staphylococcus aureus and coagulase negative staphylococci in a community and tertiary care hospital. J Clin Microbiol. 2004;42:2777-9. DOI: $10.1128 /$ JCM.42.6.27772779.2004. PMID: 15184468 .

14. HD I: Clinical microbiology procedures handbook. 2nd edition. ASM press: Washington DC; 2004.

15. Clinical Laboratory Standards Institute (CLSI) guidelines. Performance standards for antimicrobial susceptibility testing: twenty second informational supplement. CLSI document M100-S22. Clinical and Laboratory Standards Institute. Pennsylvania; Wayne; 2012.

16. Lyall Singh DK, Gupta V, Chhina D. Inducible clindamycin resistance among clinical isolates of Staphylococcus aureus. Journal of Mahatma Gandhi Institute of Medical Sciences September 2013;18(2):1125. DOI: 10.4103/0971-9903.117799.

17. Waldvogel FA: Staphylococcus aureus (including staphylococcal toxic shock). In Mandell, Douglas and Bennett's principles and practice of infectious diseases. 5th edition. Edited by Mandell GL, Bennett JE, Dolin R. Philadelphia, PA: CV, A Harcourt Health Sciences Co; 2000.

18. Laclercq R. Mechanisms of resistance to macrolides and lincosamides: Nature of resistance elements and their clinical implications. Clin Infect Dis 2002;34:482 $\square 92$. DOI:10.1086/324626. PMID:11797175. 
19. Deotale v, Mendiratta DK, Raut U, Narang P. Inducible Clindamycin Resistance in Staphylococcus aureus isolated from Clinical samples. Indian journal of medical microbiology: 2010;28:124-6. DOI: 10.4103/02550857.62488. PMID: 20404457.

20. Iqbal AF, Zeb M, Hussain A, Jahan FB, Murtuza G. Inducible Clindamycin Resistance in Staphylococcus Species. Journal of the College of Physicians and Surgeons Pakistan. 2014;24(7):481-4.

21. Roy RC, Kaundal S, Nautiyal S, Jauhari S, Mahawal BS, Khanduri A. Phenotypic detection of inducible Clindamycin resistance among Staphylococcus species. International Journal of Advanced Research. 2013;1(8):126.

22. Gunduz T, Akgul S, Ozcolpan G, Limoncu ME. Investigation of inducible clindamycin resistance among clinical isolates of Staphylococci. Afr J Microbiol Res. 2012;6:2294-8. DOI: 10.5897/AJMR11.1012.

23. Koppad M, Parameshwar S, Halesh LH, Siddesh KC. Detection of inducible clindamycin resistance in staphylococcus aureus and CONS at tertiary care hospital. Indian J Microbiol Res. 2015;2(4):192-7. DOI: 10.5958/2394-5478.2015.00012.6.

24. Ciraj AM, Vinod P, Sreejith G, Rajani K.; Inducible Clindamycin Resistance in clinical isolates of Staphylococci. Indian Journal of Pathology and Microbiology. 2009;52(1):49-51. DOI: 10.4103/03774929.44963. PMID:19136780.

25. Prabhu K, Rao S, Rao V. Inducible clindamycin resistance in Staphylococcus aureus isolated from clinical samples. J Lab Physicians. 2011;3:25-7. DOI: 10.4103/09742727.78558. PMID:21701659.

26. Fatima Khan, Sana Ali, Asfia Sultan, Meher Rizvi, Abida Khatoon, Indu Shukla, Haris M Khan. A study of inducible clindamycin resistance in erythromycin resistant clinical isolates of staphylococcus species. Asian Journal of Medical Sciences. 2015;6(6):48-52. DOI: 10.3126/ ajms.v6i6.11811.

27. Shantala GB, Shetty AS, Rahul Rao K, Vasudeva, Nagarathnamma T. Detection of Inducible Clindamycin Resistance in Clinical isolates of Staphylococcus aureus by the Disc Diffusion Induction test. Journal of Clinical and diagnostic research. 2011;5(1):35-7. 